\title{
Cost sharing, differential games, and the Moulin-Shenker rule
}

\author{
Maurice Koster $^{1}$
}

University of Amsterdam, February 2005

\begin{abstract}
The Moulin-Shenker rule (Sprumont (1998)) is a nonlinear solution concept for solving heterogeneous cost sharing problems. The first part of the paper shows an axiomatic characterization of this solution using bounds on cost shares and consistency. The second part is devoted to differential games for heterogeneous production problems. It is shown for 2-player games that by an appropriate choice of the game dynamics there is essentially a unique Markov perfect Nash equilibrium. An axiomatic analysis follows for the appropriate game dynamics, which leads in turn to a strategic characterization of the Moulin-Shenker rule.
\end{abstract}

Keywords: cost sharing, serial rule, ordinality, scale invariance, (time) consistency, cost games, differential games, Markovian (perfect) equilibrium

JEL Classification: C70, D63, D70

\section{INTRODUCTION}

This paper focuses on the problem of allocating the cost of usage of a production facility for several outputs that is jointly owned by a fixed group of agents. Each output of the technology is personal in the sense that each of the agents is interested in a different good. A cost function $c$ summarizes the relation between the level of the outputs and the minimal (monetary) input that is needed to generate this level. Given a profile of individual demands $\left(q_{1}, q_{2}, \ldots, q_{n}\right), q_{i}$ being a real number indicating the demand of agent $i$ for good $i$, we seek to distribute total costs $c\left(q_{1}, q_{2}, \ldots, q_{n}\right)$. The more eligible devices will be sensible to the level of individual demands and the cost structure. The vast majority of the

\footnotetext{
${ }^{1}$ Department of Quantitative Economics, Faculty of Economics and Econometrics, University of Amsterdam. e: mkoster@uva.nl, phone: ++31-205254226.
} 
literature on heterogeneous cost sharing problems encompasses the study of so-called $a d d i$ tive mechanisms, i.e., mechanisms that behave as linear operators with respect to the cost structure. Examples include the Aumann-Shapley mechanism (Aumann-Shapley (1974), Billera and Heath (1982)), Shapley-Shubik mechanism (Shubik (1962)), and the more recent Friedman-Moulin mechanism (Friedman and Moulin (1999), Yanovskaya (2004))). ${ }^{2}$ Examples of non-additive solutions for heterogeneous cost sharing problems are the are the ordinal proportional rule (Sprumont (1998)) the ordinally proportional rule (Sprumont (1998)), the radial serial rule (Koster et al. (1998)), and the Moulin-Shenker mechanism (Sprumont (1998)). The above cost sharing mechanisms are generalizations of mechanisms on the class of single-good or homogeneous cost sharing problems, where full comparison of the different units of the goods is legitimate, if not compelling. In this sense, the Aumann-Shapley mechanism and the ordinal proportional mechanism extend the average cost sharing mechanism (Moulin and Shenker (1994)), whereas the radial serial rule and the Moulin-Shenker mechanism generalize the serial cost sharing mechanism (Moulin and Shenker (1992), Moulin (1996)). In this paper these serial extensions are of particular interest.

The first part of the paper is devoted to an axiomatic characterization of the MoulinShenker mechanism. This mechanism seems a special candidate in the class of solutions that satisfy ordinality. This property, introduced by Sprumont (1998), requires from a solution that it does not depend on conventions used to measure an agent's demand. It therefore requires from a solution that it is invariant against essentially any transformation of measuring scales. Ordinality therefore represents a strengthening of the common property of scale invariance, that requires invariance with respect to linear transformations of scale. Examples of ordinal solutions are the Shapley-Shubik-, radial serial-, and MoulinShenker mechanism. In this respect the strength of the axiom should be stressed: the Aumann-Shapley mechanism is only scale invariant, and the Friedman-Moulin mechanism not even that. Sprumont (1998) provides very compact characterization of the MoulinShenker rule that does not include any notion of ordinality, but a property called serial principle and the rather technical partial differentiability. The serial principle extends the property independence of larger demands of the serial mechanism and seems to be the most essential feature for serial cost sharing, by which the smaller agents are protected against possibly excessive behavior of the larger demanders. However, partial differentiability lacks any intuitive interpretation. This paper presents a characterization that interchanges the serial principle with the similar self-consistency and avoids the differentiability axiom by inclusion of the following axioms: (a) scale invariance, (b) continuity, and (c) upperbound on cost shares. Continuity requires stability with respect to small changes in demands, and the upperbound property declares a maximal solidarity level for each agent in the sense that it determines a maximal price that an agent is willing to pay based on the marginal costs that are actually faced.

The second part of the paper is devoted to a simple model of integral production and

\footnotetext{
${ }^{2}$ For further references see Moulin (2002) for an overview
} 
cost sharing through differential games. It is assumed that a benevolent planner designs a game in which the agents are continuously required to state their interest in having extra production or not. Then these demands are used as inputs for an underlying system dynamics that describes how the production levels for the different goods change over time: each agent gets his extra demanded units and pays for the corresponding additional marginal cost. During the game the planner sees to it that each player is fully informed about the state of the game. Then if no agent requests more units, the game ends. The utility of an agent is non-decreasing in the level of good obtained and quasi-linear and non-increasing in the corresponding cost share. Basically the planner may infuence the outcome in the game by setting up a suitable combination of system dynamics and control spaces. This procedure mimicks the 'push the button' idea underlying the serial cost sharing rule for homogeneous technologies. ${ }^{3}$ It is assumed that the players use Markov strategies, that is, each is endowed with a fullfledged action plan that consists of an action in every contingency of the state of the game. A first result for two-player games is that for certain rules of the game, i.e. for certain combinations of dynamics and control spaces, there exists always a unique Markov perfect Nash equilibrium. It is shown that games can be defined such that the equilibrium trajectories are those underlying the Friedman-Moulin- and Moulin-Shenker cost sharing mechanism. The second result shows that the unique game dynamics that guarantees uniqueness of Markov perfect Nash equilibrium, scale invariance in equilibrium and lowerbounds on equilibrium utilities, lead to the Moulin-Shenker path as equilibrium trajectory. In particular, this shows the implementation of the Moulin-Shenker mechanism and a first strategic characterization of this solution. Moreover, for homogeneous problems this result corresponds to the strategic results of Moulin and Shenker (1992) as the equilibrium end-state in the dynamic production game corresponds to the homogeneous equilibrium quantities of the serial cost sharing mechanism.

Overview of the paper: Section 2 discusses the basic cost sharing model and formal notation. Section 3 discusses the most important solutions and key properties in this paper. Section 4 provides an axiomatic characterization of the Moulin-Shenker rule using the properties in Section 3. Section 5 introduces the basic framework for dynamic production games and discusses equilibrium properties for the special class of autonomous games. Section 6 continues by characterizing the appropriate game dynamics which leads to a characterization of the Moulin-Shenker equilibrium.

\section{The COST SHARING MODEL}

Throughout the paper we will concentrate on a fixed and finite group of agents $N=$ $\{1,2, \ldots, n\}$. The collective $N$ jointly owns some production facility for some set of goods. The output goods are personalized in the sense that there is at most one interested agent for each output. So we can speak of the set of goods $N$, where good $i \in N$ is identified

\footnotetext{
${ }^{3}$ See Moulin and Shenker (1992).
} 
with agent $i \in N$. A particular level of demanded output is then be described by a vector $q \in \mathbb{R}_{+}^{N}$, where the $i$-th coordinate $q_{i}$ is the demand of agent $i$ for good $i$. The zero element in $\mathbb{R}_{+}^{N}$ is denoted $\mathbf{0}$. Then the demand space is partially ordered by the natural ordering $\leq$. For all $q, q^{\prime} \in \mathbb{R}^{N}, q<q^{\prime}$ holds if and only if $q_{j} \leq q_{j}^{\prime}$ for all $j \in N$ with strict inequality for at least one coordinate. Whenever $q_{j}<q_{j}^{\prime}$ for all $j \in N$ then we write $q \ll q^{\prime}$. For $q^{1}, q^{2} \in \mathbb{R}^{N}$ let $q^{1} \vee q^{2}, q^{1} \wedge q^{2} \in \mathbb{R}^{N}$ be defined by $\left(q^{1} \vee q^{2}\right)_{i}=\max \left\{q_{i}^{1}, q_{i}^{2}\right\},\left(q^{1} \wedge q^{2}\right)_{i}=\min \left\{q_{i}^{1}, q_{i}^{2}\right\}$ for $i \in N$. The power set of $N$ is denoted by $\mathcal{P}(N)$. For $q \in \mathbb{R}^{N}, S \in \mathcal{P}(N), q_{S}$ is the demand profile obtained from $q$, where the demands of the players in $N \backslash S$ are set to 0 . The demand profile out of $q \in \mathbb{R}_{+}^{N}$ where the demand of a player $i$ is interchanged with $t \in \mathbb{R}_{+}$ is denoted by $\left(q^{-i}, t\right)$. The unit simplex in $\mathbb{R}^{N}$ is denoted $\Delta^{N}:=\left\{q \in \mathbb{R}_{+}^{N} \mid \sum_{i \in N} q_{i}=1\right\}$, and the Euclidean norm of a vector $q \in \mathbb{R}_{+}^{N}$ by $\|q\|$.

We assume that all information about the costs involved with bringing production up to a certain level is given by a cost function $c: \mathbb{R}_{+}^{N} \rightarrow \mathbb{R}_{+}$. In this paper we will only be concerned with cost functions $c$ that are continuously differentiable and increasing, i.e. if $x<y$ then $c(x)<c(y)$. Moreover the partial derivatives of $c, D_{i} c$, are supposed to be Lipschitz continuous and bounded away from 0 and $\infty$, i.e. there are $a(c), b(c)>0$ such that for all $x \in \mathbb{R}_{+}^{N}$

$$
a(c) \leq D_{i} c(x) \leq b(c) \text { for all } i \in N \text {. }
$$

In addition there are no fixed costs, which amounts to the condition $c(\mathbf{0})=0$. The class of all such cost functions is denoted by $\mathcal{C} ; \mathcal{C}^{+}$is the set of convex cost functions in $\mathcal{C}$ and $\mathcal{C}^{++} \subseteq \mathcal{C}^{+}$consists of all strictly convex elements. A cost function $c \in \mathcal{C}$ is called normalized if $D_{i} c(\mathbf{0})=1$ for all $i \in N$.

A cost sharing problem is an ordered pair $(q, c) \in \mathbb{R}_{+}^{N} \times \mathcal{C}$. The class of all cost sharing problems is denoted by $\mathcal{G}$. A cost sharing rule or cost sharing mechanism is a mapping $\mu: \mathcal{G} \rightarrow \mathbb{R}_{+}^{N}$ associating to each cost sharing problem $(q, c)$ an efficient vector of cost shares $\mu(q, c) \in \mathbb{R}_{+}^{N}$, i.e. $\sum_{i \in N} \mu_{i}(q, c)=c(q)$.

\section{Cost Sharing Mechanisms And PROPERTies}

The basic distinction between the heterogeneous and homogeneous models is that in the first one asymmetries in the cost function may arise. Most eligible cost sharing mechanisms in the literature treat these asymmetries by relating an agents cost share to some measurement of related marginal costs. For example, the Aumann-Shapley mechanism calculates average marginal costs along the diagonal path towards the final production level. The Friedman-Moulin serial extension determines cost shares for $(q, c)$ through measuring the marginal cost along the path $\gamma^{q}$ defined by $\gamma^{q}(t)=t e_{N} \wedge q$ for all $t \in \mathbb{R}_{+}$, such that agent $i \in N$ is charged

$$
\mu_{i}^{\mathrm{FM}}(q, c)=\int_{0}^{\infty} D_{i} c\left(\gamma^{q}(t)\right)\left(\gamma^{q}\right)_{i}^{\prime}(t) \mathrm{d} t
$$


When the argument of $\gamma^{q}$ is seen as a time parameter, it can be considered to describe a real-time production process.

Now consider $(q, c) \in \mathcal{G}$ and the the system of partial differential equations

$$
D_{i} \gamma(t)= \begin{cases}\frac{1}{D_{i} c(\gamma(t))} & \text { if } t \geq 0 \text { and } \gamma_{i}(t)<q_{i}, \\ 0 & \text { else. }\end{cases}
$$

It can be shown that there is a unique solution $\gamma^{q, c}$ to these equalities such that $\gamma^{q, c}(0)=\mathbf{0}$. Essentially, this is due to Lipschitz continuity of each $1 / D_{i} c$ together with condition (1), see Coddington and Levinson (1955)). ${ }^{4}$ Needless to say that this curve $\gamma^{q, c}$ heavily depends on the demand profile $q$ and the cost function $c \in \mathcal{C}$. The solution $\gamma^{q, c}$ can be interpreted as a production device. Suppose that the intensity at which an agent $i$ is served by means of the plan $\gamma^{q, c}$ at moment $t$ is measured by the corresponding marginal $\operatorname{cost} D_{i} c\left(\gamma^{q, c}(t)\right) D_{i} \gamma^{q, c}(t)$. Then $\gamma^{q, c}$ can be intuitively interpreted as a device by which goods are distributed with equal intensity for those agents that are not fully served at $t$, since for those agents $i$, it holds $D_{i} c\left(\gamma^{q, c}(t)\right) D_{i} \gamma^{q, c}(t)=1$. The Moulin-Shenker rule now charges agent $i$ for the marginal costs $D_{i} c$ along the production device $\gamma^{q, c}$ in the cost sharing problem $(q, c)$.

Definition 3.1 Let $(q, c) \in \mathcal{G}$. The Moulin-Shenker rule $\mu^{\mathrm{MS}}$ determines the individual cost shares by taking the integral of all marginal cost along the curve $\gamma^{q, c}$, which solves the above system of differential equations (2). Then for all $i \in N$,

$$
\mu_{i}^{\mathrm{MS}}(q, c)=\int_{0}^{\infty} D_{i} c\left(\gamma^{q, c}(s)\right) D_{i} \gamma^{q, c}(s) d s .
$$

Example 3.2 Consider the cost sharing problem $(q, c)$ with $N=\{1,2\}, q=(10,10)$, and $c$ is the cost function on the block $B=[0,(15,15)]$ defined by $c\left(t_{1}, t_{2}\right)=e^{2 t_{1}+t_{2}}-1$. In order to determine the cost shares $\mu_{1}^{\mathrm{MS}}(q, c)$ en $\mu_{2}^{\mathrm{MS}}(q, c)$ we first solve the differential equation (2). We calculate the partial derivatives on $B$,

$$
D_{1} c\left(t_{1}, t_{2}\right)=2 e^{2 t_{1}+t_{2}}=2 D_{2} c\left(t_{1}, t_{2}\right) \text { for all }\left(t_{1}, t_{2}\right) \in B .
$$

So according to $\gamma^{q, c}$, until one of the demands is reached, twice as much of good 2 is produced relative to the production of good 1. So $\gamma^{q, c}$ can be taken such that

$$
\gamma^{q, c}(t)= \begin{cases}(t, 2 t) & \text { if } t \leq 5 \\ (t, 10) & \text { for } 5<t \leq 10\end{cases}
$$

Then this amounts to the cost shares

$$
\begin{aligned}
& \mu_{2}^{\mathrm{MS}}(q, c)=\frac{1}{2} c\left(\gamma^{q, c}(5)\right)=\frac{1}{2} c(5,10)=\frac{1}{2}\left(e^{20}-1\right), \\
& \mu_{1}^{\mathrm{MS}}(q, c)=\mu_{2}^{\mathrm{MS}}(q, c)+c\left(\gamma^{q, c}(10)\right)-c\left(\gamma^{q, c}(5)\right)=e^{30}-\frac{1}{2}\left(e^{20}+1\right) .
\end{aligned}
$$

\footnotetext{
${ }^{4}$ Just take $K$ such that $\mid D_{i} c(x)-D_{i} c(y) \leq K\|x-y\|$. Then $\left|\left(D_{i} c(x)\right)^{-1}-\left(D_{i} c(y)\right)^{-1}\right| \leq K a(c)^{-2}\|x-y\|$.
} 
Sprumont (1998) argues that cost sharing mechanisms should not depend on conventions used to measure the individual demands. This is expressed by the notion of ordinality, that requires that a cost sharing mechanism is invariant under all ordinal transformations of the cost sharing problem. For our purposes, we need only the more familiar and weaker notion of scale invariance, which imposes independence of rescaling units of the measuring scales. For instance, if the output of some good is measured by weight, scale invariance tells us that the cost shares should not depend on the fact that we expressed the amounts in kilos instead of tons. We like to stress the gap between ordinality and scale invariance: ordinality requires also invariance with respect to all non-linear increasing transformations of the measuring scales.

Definition 3.3 A function $f: \mathbb{R}_{+}^{N} \rightarrow \mathbb{R}_{+}^{N}$ is a positive linear transformation of scales if there is $\alpha \in \mathbb{R}_{++}^{N}$ such that $f(y)=\left(\alpha_{1} y_{1}, \ldots, \alpha_{n} y_{n}\right)$ for all $y \in \mathbb{R}_{+}^{N}$. A cost sharing mechanism $\mu$ is scale invariant (SI) if for all such mappings $f$ and all cost sharing problems $(q, c) \in \mathcal{G}$ it holds that $\mu(q, c)=\mu\left(f(q), c \circ f^{-1}\right)$.

In the subsequent analysis a minimum stability from mechanisms is required, which is formalized by continuity, that requires robustness of a rule with respect to small changes in the data that is used to model the cost sharing problem. To be precise, small perturbations of the demand profile should not result in large changes in the cost shares:

Definition 3.4 A cost sharing mechanism $\mu$ is continuous (CONT) if for all $c \in \mathcal{C}$, the mapping $q \mapsto \mu(q, c)$ is continuous on $\mathbb{R}_{+}^{N}$.

Unequal cost shares point at the fact that agents are not considered to be symmetric in the cost sharing problem at hand. The size of a demand and the cost structure play an essential role here. Intuitively it makes sense to judge a good more expensive than another if the marginal costs of the first are higher than the other, always. The more eligible cost sharing mechanisms are fully consistent with this idea in the sense that the cost share of an agent increases with the corresponding marginal costs. In this way the marginal cost functions are taken as benchmarks in judging asymmetries in cost sharing problems. If this is taken as a starting point, one could ask to what extent cost shares should relate to the marginal costs. This leads us to focus on the following property, that requires that an agent never pays more than the related marginal cost function could support.

Definition 3.5 A cost sharing mechanism $\mu$ satisfies upperbound (UPP) if for all cost sharing problems $(q, c) \in \mathcal{G}$ it holds that for all $i \in N$

$$
\mu_{i}(q, c) \leq q_{i} \max _{y \in[\mathbf{0}, q]} D_{i} c(y) .
$$


Note that the maximum over $[\mathbf{0}, q]$ exists by continuity of the partial derivatives. Bounds on cost shares are also discussed in the literature on homogeneous literature, see e.g. Koster (2002) and Hougaard and Petersen (2001).

Though UPP can be considered weak as a characterizing property, it is strong enough to imply other more frequently used properties:

(1) UPP implies no exploitation, i.e. zero demanders pay nothing, since $q_{i}=0$ implies that $\mu_{i}(q, c) \leq \max _{y \in[\mathbf{0}, q]} D_{i} c(y)=0$ and $\mu_{i}(q, c) \geq 0$.

(2) UPP implies linearity, i.e. for linear cost functions the cost shares of the agents are linear in demand. To be more precise, if $c \in \mathcal{C}$ on $[\mathbf{0}, q]$ is given by $c(y)=\sum_{i \in N} \alpha_{i} y_{i}$ for some $\alpha \in \mathbb{R}_{++}^{N}$, then UPP implies $\mu_{i}(q, c)=\alpha_{i} q_{i}$ for all $i \in N$. The next lemma essentially shows that if a problem is almost linear, and even homogeneously so, then under UPP the cost sharing mechanism behaves almost linearly. A proof is found in the appendix.

Lemma 3.6 Consider a cost sharing problem $(q, c) \in \mathcal{G}$ with $D_{i} c(\mathbf{0})=1$ for all $i \in N$. Then for a cost sharing mechanism $\mu$ with the property UPP it holds that

$$
\lim _{t \downarrow 0} \frac{\mu_{i}\left(t e_{N}, c\right)}{c\left(t e_{N}\right)}=\frac{1}{|N|}
$$

where $e_{N} \in \mathbb{R}_{+}^{N}$ is the vector with all coordinates 1 .

In the next section, the Moulin-Shenker cost sharing mechanism will be singled out from a class of cost sharing mechanisms that have the serial principle in common. The serial principle prevents the smaller agent to get overexposed to the consequences of contingent excessive behavior of other agents. This principle was formulated for the homogeneous case by Moulin and Shenker (1992a) as independence of size of larger demands. The corresponding formulation makes use of intercomparison of individual demands. But, typically, the heterogeneous model case lacks a natural way of ordering demands such that they can be compared in a direct way. Still, if a mechanism is singled out for some fairness properties, then there is just one consistent way of comparing the demands, and that is by comparing the size of the corresponding cost shares for the problem at hand. The serial principle then urges that once the mechanism values the demand of an agent $i$ lower than that of agent $j$, any further increase of agent $j$ 's demand should have no effect at all on agent $i$ 's cost share. This idea is due to Sprumont (1998). Formally, A cost sharing mechanism satisfies the serial principle if for all cost sharing problems $(q, c) \in \mathcal{G}$ it holds that for all $i \in N$ and $j \in N \backslash\{i\}$ with $\mu_{j}(q, c) \geq \mu_{i}(q, c)$ it holds that for $t \geq q_{j}$, $\mu_{i}\left(\left(q^{-j}, t\right), c\right)=\mu_{i}(q, c)$.

In general, though the names are quite suggestive, being a serial extension is not sufficient for a method to satisfy the serial principle. The Friedman-Moulin rule illustrates this distinction; it is a serial extension only. 
Note that in our setting the serial principle implies positivity, i.e. $q_{i}>0$ implies $\mu_{i}(q, c)>0$ for all $i \in N$ and all problems $(q, c) \in \mathcal{G}$. For every non-positive mechanism possibly free-riders enter the picture. Any increase of any agent's demand causes a rise of total cost, so the impact on total cost of any non-zero demander is considered to be positive. Consequently, positivity can be considered as compelling for our purposes.

It is easily seen that for every positive cost sharing mechanism the content of (5) is exactly rendered, for all normalized cost functions $c \in \mathcal{C}$, by

$$
\lim _{t \downarrow 0} \frac{\mu_{i}\left(t e_{N}, c\right)}{\mu_{j}\left(t e_{N}, c\right)}=1 \text { for all } i, j \in N .
$$

Moulin and Shenker (1992) discuss the property free lunch which combines a mild form of justice with a weak form of consistency (see also Kolpin (1994) and Thomson (1990, 1995)). To generalize this idea we develop the notion of self-consistency. This notion makes it possible to link outcomes for problems of different size.

Essentially, a cost sharing mechanism is used as an instrument of evaluation; the agent with the larger cost share can be considered to have a larger demand. In this way, for a problem $(q, c)$, all the demands are equally valued by a cost sharing mechanism $\mu$ if and only if $\mu_{i}(q, c)=\mu_{j}(q, c)$ for all $i, j \in N$.

Fix a cost sharing problem $(q, c)$ and a cost sharing mechanism $\mu$. Suppose that we provide all agents with equally valued parts of their demands; agent $i$ gets $d_{i} \leq q_{i}$ such that $\mu_{i}(d, c)=\mu_{j}(d, c)$ for all $i, j \in N$. Then the reduced cost sharing problem is defined by the profile of unfulfilled demands $q-d$, and the cost data for any level of production beyond $d$ as is summarized by $c^{d}$. Now self-consistency allows for determining the final cost shares by independently solving the problems $(d, c)$ and $\left(q-d, c^{d}\right)$ and taking the sum over the corresponding outcomes.

In the same spirit we deal with those situations where there are some zero demanders. It is reasonable to require that just their presence should have no effect on the allocation of costs for the other agents. Suppose again that $d$ is a demand profile smaller than $q$, such that the non-zero demanders are equally evaluated by the mechanism $\mu$. Then selfconsistency proposes $\mu(q, c)_{S}=\mu(d, c)_{S}+\mu\left(q-d, c^{d}\right)_{S}$, where $S$ is the set of the non-zero demanders for $q$. So, if cost shares differ, then this is not due to the part of the problem that the agents are equally charged for, but due to asymmetries in the related reduced problem.

Definition 3.7 A cost sharing mechanism $\mu$ satisfies self-consistency (SCONS) if for all cost sharing problems $(q, c) \in \mathcal{G}$ such that $q_{N \backslash S}=\mathbf{0}_{N \backslash S}$ for some $S \in \mathcal{P}(N)$ and $d \leq q$ such that $\mu_{i}(d, c)=\mu_{j}(d, c)$ for all $i, j \in S$,

$$
\mu(q, c)_{S}=\mu(d, c)_{S}+\mu\left(q-d, c^{d}\right)_{S}
$$




\section{Characterization of the Moulin-Shenker Rule}

Next we will define the class of path generated cost sharing methods. The idea is in fact adopted from Sprumont (1998), but notations are different.

For $S \subseteq N$ a path in $\mathbb{R}_{+}^{S}$ is a continuous mapping $\pi: \mathbb{R}_{+} \rightarrow \mathbb{R}_{+}^{S}$ with $\pi(0)=0$. The path $\pi$ is increasing if $\pi_{i}(t)<\pi_{i}\left(t^{\prime}\right)$ for all $i \in S$ if only $t<t^{\prime}$. In our setting, with the argument of $\pi$ thought of as being time, an increasing path may be considered as a program for production. At time $t$ an amount of good $i$ equal to $\pi_{i}(t)$ units is produced for agent $i$.

Suppose that for each pair $(d, c) \in \mathcal{G}$ we have an increasing path $\pi^{d, c, S}$ for $S$ such that for each $q \in \mathbb{R}_{+}^{S}$ there is $t \in \mathbb{R}_{+}$with $\pi^{d, c, S}(t)>q$. Such a path will be considered to describe a fictitious production plan for coalition $S$ from level $d \in \mathbb{R}_{+}^{N}$. Possibly such a plan will depend on the exogenous information of costs that is summarized by $c \in \mathcal{C}$.

$\Pi$ is defined as the collection of all those paths, one for each triple $(d, c, S) \in \mathbb{R}_{+}^{N} \times \mathcal{C} \times \mathcal{P}(N)$. We will refer to $\Pi$ as a path collection. A path collection $\Pi$ defines for each cost sharing problem $(q, c) \in \mathcal{G}$ a production plan, casu quo a path $\pi$ for $N$ in the following way.

We start at production level 0 . Initially, we take the path for $N, \pi^{0, c, N}$ as a production device, telling us for each moment in time what is produced for the individual agents. So follow $\pi^{0, c, N}$ up to the earliest moment $t_{1}$ that some agents $M_{1} \subseteq N$ are satisfied, i.e.

$$
\pi_{i}^{0, c, N}\left(t_{1}\right)=q_{i} \text { for all } i \in M_{1} \text {. }
$$

Define $\pi$ on $\left[0, t_{1}\right]$ by $\pi(t)=\pi^{0, c, N}(t)$. Let $d^{1}$ denote the total demand that is processed so far, $d^{1}=\pi\left(t_{1}\right)$. Still, an agent $i \in N \backslash M_{1}$ needs $q_{i}-d_{i}^{1}$ units of good $i$ in order to be satisfied. Next, we take $\pi^{d^{1}, c, N \backslash M_{1}}$ as the additional production plan for $N \backslash M_{1}$ until the first moment $t_{2}$ that some agents $M_{2} \subseteq N \backslash M_{1}$ are satisfied, i.e.

$$
\pi^{d^{1}, c, N \backslash M_{1}}\left(t_{2}\right)=q_{i}-d_{i}^{1} \text { for all } i \in M_{2} .
$$

The definition of $\pi$ is now completed up to moment $t_{1}+t_{2}$ by

$$
\pi\left(t+t_{1}\right):=d^{1}+\left(0_{M_{1}}, \pi^{d^{1}, c, N \backslash M_{1}}(t)\right) \text { for all } t \in\left(0, t_{2}\right] .
$$

Let $d^{2}=\pi\left(t_{1}+t_{2}\right)$. Follow the production device $\pi^{d^{2}, c, N \backslash\left(M_{1} \cup M_{2}\right)}$ until moment $t_{3}$ where the first agents $M_{3} \subseteq N \backslash\left(M_{1} \cup M_{2}\right)$ are fulfilled with their remaining needs $q_{M_{3}}-d_{M_{3}}^{2}$. Then define, for all $t \in\left(0, t_{3}\right]$,

$$
\pi\left(t+t_{1}+t_{2}\right)=d^{2}+\left(0_{M_{1} \cup M_{2}}, \pi^{d^{2}, c, N \backslash\left(M_{1} \cup M_{2}\right)}(t)\right) .
$$

In this way we can go on and complete the definition of $\pi$. We just proceed by determining time levels $t_{4}, t_{5}, \ldots$ and corresponding groups of agents $M_{4}, M_{5}, \ldots$ until the first moment $t_{1}+\ldots+t_{k}$ such that there are no remaining demanders, i.e. $N \backslash\left(M_{1} \cup \ldots \cup M_{k}\right)=\emptyset$. Note that $\pi(t)=q$ when $t>t_{1}+t_{2}+\ldots+t_{k}$. We will say that $\pi$ is the path for $(q, c)$ generated by $\Pi$.

Definition 4.1 The solution for the cost sharing problem $(q, c) \in \mathcal{G}$ generated by a path collection $\Pi$ is the vector $\mu^{\Pi}(q, c) \in \mathbb{R}_{+}^{N}$ defined as follows. Let $\pi$ be the path for $(q, c)$ 
generated by $\Pi$. Suppose that according to $\pi$ agent $i$ is satisfied at moment $t_{i}$. Without loss of generality, assume that $t_{i} \leq t_{j}$ whenever $i \leq j$ for all $i, j \in N$. We split the successive cost increments $c\left(\pi\left(t_{i+1}\right)\right)-c\left(\pi\left(t_{i}\right)\right)$ equally among the agents requiring service on the interval $\left(t_{i}, t_{i+1}\right]$. By assumption this is the set of agents $\{i+1, i+2, \ldots, n\}$. Then this boils down to $\mu_{1}^{\Pi}(q, c)=\frac{c\left(\pi\left(t_{1}\right)\right)}{N}$ as the cost share for agent 1 , while the cost shares for the other agents $i \in N$ are inductively defined through

$$
\mu_{i}^{\Pi}(q, c)=\mu_{i-1}^{\Pi}(q, c)+\frac{c\left(\pi\left(t_{i}\right)\right)-c\left(\pi\left(t_{i-1}\right)\right)}{n-i+1} .
$$

By varying over all cost sharing problems in $\mathcal{G}$ this yields a cost sharing mechanism $\mu^{\Pi}$, generated by the path collection $\Pi$.

We will also say that in the above definition the cost shares for the problem $(q, c)$ are generated by $\Pi$. Note that for a path generated method only the images of the paths count, irrespective of the parametrization. Keeping this in mind, one should have no problem with the following.

Lemma 4.2 Let $f: \mathbb{R}_{+}^{N} \rightarrow \mathbb{R}^{N}$ be a linear transformation of measuring scales. Suppose $\mu$ is a scale invariant cost sharing mechanism that is generated by a path collection $\Pi=\left\{\pi^{d, c, S} \mid(d, c, S) \in \mathbb{R} \times \mathcal{C} \times \mathcal{P}(N)\right\}$. If the cost shares for $(q, c) \in \mathcal{G}$ are generated by $\pi$, then the cost shares for $\left(f^{-1}(q), c \circ f\right)$ are generated by $f^{-1} \circ \pi$.

A simple but important observation is that each path generated method indeed satisfies the serial principle. Sprumont (1998) proves the converse of this statement for all continuous mechanisms. The next lemma is similar, its proof is rather lengthy postponed till the appendix.

Lemma 4.3 A continuous cost sharing mechanism satisfies no exploitation and self consistency only if it is generated by a collection of paths.

Especially, Lemma 4.3 shows that a continuous cost sharing mechanism with the properties self consistency and no exploitation satisfies the serial principle. However, self consistency is fundamentally different from the serial principle. For instance, it is easy to define path generated cost sharing rules, that satisfy no exploitation and continuity and fail to obey self consistency. Furthermore, splitting cost equally for all cost sharing problems defines a self consistent rule that neither satisfies no exploitation nor the serial principle.

Fix a cost function $c \in \mathcal{C}$. For each $d \in \mathbb{R}_{+}^{N}$, let $c^{d} \in \mathcal{C}$ be the cost function that relates each increase of demand $q$ after $d$ to the corresponding incremental cost, i.e. $c^{d}(q)=$ $c(d+q)-c(q)$ for all $q \in \mathbb{R}^{N}$. An ordered pair $(d, S) \in \mathbb{R}_{+}^{N} \times \mathcal{P}(N)$ gives rise to a system of differential equations like equation (2). Let $\gamma: \mathbb{R}_{+} \rightarrow \mathbb{R}_{+}^{N}$ be such that for all $t \in \mathbb{R}_{+}$ 
and all $i \in S$

$$
D_{i} \gamma(t)=\frac{1}{D_{i} c^{d}(\gamma(t))}
$$

By the regularity assumptions on $c$ this system has a unique solution, which we will denote by $\gamma^{d, c, S}$. Then by varying over all triples $(d, c, S) \in \mathbb{R}_{+}^{N} \times \mathcal{C} \times \mathcal{P}(N)$ this gives rise to a path collection $\Gamma$, which in turn generates the Moulin-Shenker rule. Note that $\gamma^{d, c, S}=\gamma^{0, c^{d}, S}$ for all $(d, c, S) \in \mathbb{R}_{+}^{N} \times \mathcal{C} \times \mathcal{P}(N)$.

Sprumont (1998) shows that among the class of all path generated methods there is only one for which all partial derivatives with respect to the demand input exist, and that is $\mu^{\mathrm{MS}}$. Actually, it can be shown that $\mu^{\mathrm{MS}}$ is continuously differentiable.

We are now ready for the main result in this section.

Theorem 4.4 There is only one continuous, self consistent and scale invariant cost sharing mechanism that satisfies no exploitation and equation (5), and that is the MoulinShenker rule.

Corollary 4.5 There is only one continuous, self consistent and scale invariant cost sharing mechanism that satisfies upperbounds, and that is the Moulin-Shenker rule.

Proof. This implied by Lemma 3.6.

At this point it is important to realize that the above proofs suffice for a full characterization of $\mu^{\mathrm{MS}}$ on the classes $\mathcal{G}^{+}$or $\mathcal{G}^{++}$. The basic operations that are used are linear transforms which keep the nature of the problem intact.

\section{Dynamic Cost Sharing Games}

For homogeneous good models there are results concerning cost sharing games - see Moulin and Shenker (1992), Moulin (1996), Kolpin (1996). These games are all one-shot games. For heterogeneous good models we present a cost sharing game using a differential game, i.e., a dynamic game in continuous time. The agents are players in the dynamic game whose actions influence a dynamical system that in turn determines a production path. The game ends when no player has a request for additional production. Then each obtains the corresponding end-state production level of his good and he has to pay for the total of marginal costs along the production path.

We will make the following assumptions:

- The cost functions are chosen from $\mathcal{C}^{++}$. 
- Each agent is endowed with quasi-linear preferences over combinations of amounts of good $Y_{i}$ and costs. Then these preferences are summarized by utility functions $\left\{H_{i}\right\}_{i \in N}$ such that

$$
H_{i}\left(q_{i}, c_{i}\right)=F_{i}\left(q_{i}\right)-c_{i},
$$

for some non-decreasing function $F_{i}$. Moreover, we will assume that $F_{i}$ is differentiable and concave, so that the function $f_{i}=F_{i}^{\prime}$ is continuous and nonincreasing. In particular, it is assumed that the preferences of the players are time-independent.

The state of the game is described by a vector $x(t) \in \mathbb{R}_{+}^{N}$ which describes the total production for each agent at time $t ; x_{i}(t)$ is the amount of good $Y_{i}$ that has been produced for agent $i$ by time $t$. Suppose that $x\left(t^{0}\right)=x^{0}$ for some $t^{0} \in \mathbb{R}_{+}$. The non-decreasing path $t \mapsto x(t)$ is finite if it locks in, i.e. there exists $x \in \mathbb{R}_{+}^{N}$ such that $\lim _{t \rightarrow \infty} x(t)=x^{*}$. Then the utility of player $i$ along such $x$ is defined as

$$
J_{i}(x)=\int_{t^{0}}^{\infty}\left(f_{i}\left(x_{i}(t)\right)-D_{i} c(x(t))\right) \dot{x}_{i}(t) \mathrm{d} t .
$$

Define the profitable region for player $i$ as the set $R_{i}=\left\{x \in \mathbb{R}_{+}^{2} \mid f_{i}\left(x_{i}\right) \geq D_{i} c(x)\right\}$. A strategy of a player $i \in N$ in $\Gamma_{g, c}\left(x^{0}, t^{0}\right)$ is a mapping $u_{i}$ that assigns to each pair $(x, t) \geq\left(x^{0}, t^{0}\right)$ a real number $u_{i}(x, t) \in \mathbb{R}$ from his set of feasible controls $U_{i}(x, t, c) \subseteq \mathbb{R}_{+}$. Denote the set of all strategies of player $i$ by $S_{i}$, and let $S=\times_{i \in N} S_{i}$. Depending on the state variable and the players' control variables the system evolves via a set of differential equations

$$
\dot{x}(t)=g(x(t), u(x(t), t), t), x\left(t^{0}\right)=x^{0},
$$

where the system dynamics $g$ is defined as a mapping $\Omega \rightarrow \mathbb{R}_{+}^{N}$ with

$$
\Omega=\left\{(x, u, t) \mid x \in X, t \in\left[t^{0}, \infty\right), u \in U(x, t, c)\right\} .
$$

Given the strategy profile $u$ the corresponding utility for player $i$ is defined as $\mathcal{J}_{i}(u)=$ $J_{i}(x)$, where $x$ is the (unique) solution to (8). The tuple

$$
\Gamma_{g, c}\left(x^{0}, t^{0}\right)=\left\langle N,\left\{S_{i}\right\}_{i \in N},\left\{\mathcal{J}_{i}\right\}_{i \in N}\right\rangle
$$

is referred to as a dynamic production game at $\left(x^{0}, t^{0}\right)$. Then $\Gamma_{g, c}\left(x^{0}, t^{0}\right)$ is seen as subgame of $\Gamma_{g, c}(\mathbf{0}, 0)$. A game $\Gamma_{g, c}\left(x^{0}, t^{0}\right)$ is called autonomous if $g_{i}(x, u, t)=u_{i}$ for all $i \in N$ and where the strategy spaces are determined for $c \in \mathcal{C}$ via a Lipschitz-continuous function $h^{c}: \mathbb{R}_{+}^{N} \rightarrow \mathbb{R}_{+}^{N}$ such that the control spaces are given by $U_{i}(x, t, c)=\left[0, h_{i}^{c}(x)\right]$ for all $i \in N$. Denote the family $\left\{h^{c}\right\}_{c \in \mathcal{C}}$ by $h$. We will subsequently be concerned with a fixed $h$, and use $\Gamma_{h, c}\left(x^{0}, t^{0}\right)$ to denote the autonomous game at $\left(x^{0}, t^{0}\right)$ with cost function $c$ and control spaces that are determined by $h^{c}$.

A strategy profile $\left(\psi_{1}, \ldots, \psi_{n}\right)$ is called a Markovian Nash equilibrium for $\Gamma_{g, c}\left(x^{0}, t^{0}\right)$ if for all $i \in N$ the corresponding objective function $u_{i} \mapsto \mathcal{J}_{i}\left(\left(\psi^{-i}, u_{i}\right)\right)$ is maximized at $u_{i}=\psi_{i}$. 
In addition, the strategy profile $\left(\psi_{1}, \ldots, \psi_{n}\right)$ is called a Markov perfect Nash equilibrium for $\Gamma_{g, c}(\mathbf{0}, 0)$ if it induces a Markov Nash equilibrium for each subgame $\Gamma_{g, c}\left(x^{0}, t^{0}\right)$.

Theorem 5.1 Take an autonomous game $\Gamma_{h, c}(\mathbf{0}, 0)=\left\langle\{1,2\},\left\{S_{1}, S_{2}\right\},\left\{\mathcal{J}_{1}, \mathcal{J}_{2}\right\}\right\rangle$ and suppose that the system of differential equations specified by $\dot{x}(t)=h^{c}(x(t))$ has a unique solution for each initial value $x\left(t^{0}\right)=x^{0}$. Then Markov perfect equilibria in $\Gamma_{h, c}(\mathbf{0}, 0)$ exist, and each is represented by the tuple $\left(\psi_{1}, \psi_{2}\right)$

$$
\psi_{i}(x, t)= \begin{cases}h_{i}^{c}(x) & \text { if } x \in R_{i} \\ 0 & \text { else. }\end{cases}
$$

Proof. Note that the proof is obvious in case $f_{i}(0) \leq D_{i} c(\mathbf{0})$ for some $i$. So assume that the interior of $R_{1} \cap R_{2}$ is nonempty. The pair of strategies $\psi=\left(\psi_{1}, \psi_{2}\right) \in S_{1} \times S_{2}$ uniquely defines a path $p^{*}$ with some end-state $x^{*}$ in the boundary of $R_{1} \cap R_{2}$. By assumption on $h$ the end-state production quantities $x_{i}^{*}$ are reached by $p^{*}$ at times $t_{1}^{*}$ and $t_{2}^{*}$ and $p^{*}\left(t_{1}^{*} \vee t_{2}^{*}\right)=x^{*}$ Since $g_{i}(x(t), u(x(t), t), t)=h_{i}^{c}(x(t))>0$ for each $i$ the coordinate mapping $p_{i}^{*}$ is strictly increasing on $\left[0, t_{i}^{*}\right]$. Suppose that player $i$ adopts a strategy $\varphi_{i}$ instead of $\psi_{i}$ and assume without loss of generality that the pair of strategies $\left(\psi^{-i}, \varphi_{i}\right)$ leads to the solution path $\bar{p}$. From (10) we conclude that $\bar{p}$ has an end-state $\bar{x} \in R$. By convexity of $c$ and concavity of $F_{i}$, the equations $D_{i} c(x)=f_{i}\left(x_{i}\right)$ define $x_{i}$ as a decreasing function $w_{i}$ of $x^{-i}, x_{i}=w_{i}\left(x^{-i}\right)$. Then this yields in combination with (10) $\bar{x}_{i} \leq x_{i}^{*}$. A graphical representation is given by Figure 1 . Let $\bar{t}$ be the unique solution to $p_{i}^{*}(t)=\bar{x}_{i}$ and $\alpha: \mathbb{R}_{+} \rightarrow \mathbb{R}_{+}$such that $\bar{p}_{i}(\alpha(t))=p_{i}^{*}(t)$ for all $t \in[0, \bar{t}]$. Then first of all

$$
\begin{aligned}
\mathcal{J}_{i}\left(\psi_{1}, \psi_{2}\right) & =J_{i}\left(p^{*}\right)=F_{i}\left(x_{i}^{*}\right)-\int_{0}^{t_{i}^{*}} D_{i} c\left(p^{*}(t)\right) \cdot\left(p^{*}\right)_{i}^{\prime}(t) \mathrm{d} t \\
& \geq F_{i}\left(\bar{x}_{i}\right)-\int_{0}^{\bar{t}} D_{i} c\left(p^{*}(t)\right) \cdot\left(p^{*}\right)_{i}^{\prime}(t) \mathrm{d} t \\
& =F_{i}\left(\bar{x}_{i}\right)-\int_{0}^{\bar{t}} D_{i} c\left(p^{*}(t)\right) \cdot(\bar{p} \circ \alpha)_{i}^{\prime}(t) \mathrm{d} t
\end{aligned}
$$

But then continue by using the stict convexity of $c$ in order to obtain

$$
\begin{aligned}
\mathcal{J}_{i}\left(\psi_{1}, \psi_{2}\right) & >F_{i}\left(\bar{x}_{i}\right)-\int_{0}^{\bar{t}} D_{i} c(\bar{p} \circ \alpha(t)) \cdot(\bar{p} \circ \alpha)_{i}^{\prime}(t) \mathrm{d} t \\
& =J_{i}(\bar{p} \circ \alpha)=J_{i}(\bar{p})=\mathcal{J}_{i}\left(\psi^{-i}, \varphi_{i}\right) .
\end{aligned}
$$

This shows basically that the optimal strategy of a player $i$ is to choose his control as high as possible on his profitable region $R_{i}$. A similar reasoning shows that in each subgame the players perform optimally only when they play with maximal control levels. By assumption on $h$ a unique solution path for the system of differential equations with arbitrary starting point is assured and the earlier reasoning applies here as well to show 


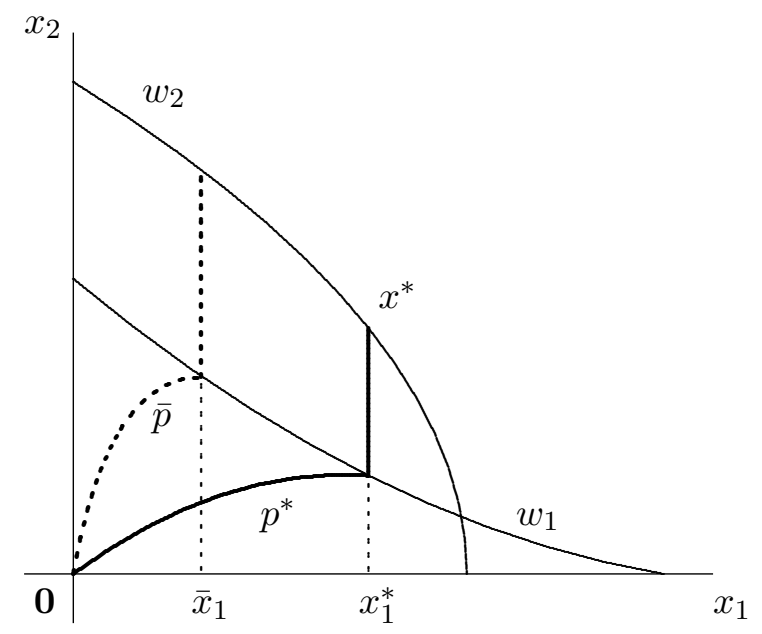

FiguRE 1

that the formula (10) specifies a Markov Nash equilibrium in the subgame. Finally, assume that there is some equilibrium other than is specified by (10). Then there is a subgame where some player $i$ did not choose his controls maximal almost everywhere along the equilibrium trajectory. The reasoning in the first part of the proof applies here as well in order to show that $i$ will increase his payoff in this particular subgame by increasing his controls. Obviously, this contradicts the equilibrium property.

Example 5.2 Consider $h=\left\{h^{c}\right\}_{c \in \mathcal{C}}$ with $h_{i}^{c}(x)=1 / D_{i} c(x)$. Then Theorem 5.1 shows that in the corresponding Markov perfect Nash Equilibrium each player $i$ chooses his control equal to $1 / D_{i} c(x)$ as long as marginal costs for his good $Y_{i}$ are lower than marginal profits. If the end-state is $q^{*}$, then in equilibrium each player pays $\mu^{\mathrm{MS}}\left(q^{*}, c\right)$.

Example 5.3 Consider $h=\left\{h^{c}\right\}_{c \in \mathcal{C}}$ with $h^{c}(x)=e_{N}$. The corresponding equilibrium specifies an equilibrium trajectory that equals the Friedman-Moulin path and if the endstate is $q^{*}$ then the profile of cost shares for the players equals the Friedman-Moulin cost shares.

Remark The above theorem can significantly be generalized. For the game dynamics $g$ we only need to assume that for any $c \in \mathcal{C}^{++}$it holds that the mapping $\left.u_{i} \mapsto g_{(} x,\left(u^{-i}, u_{i}\right), t\right)$ is monotonically increasing and Lipschitz continuous. Since in that case we can define $\tilde{h}^{c}(x, t)=g(x, h(x, t), t)$ and start Theorem 5.1 with $h^{c}=\tilde{h}^{c}$. 


\section{Designing the APpropriate Game Dynamics}

A benevolent planner may use a dynamic production game as a tool to solve the integrated production and cost sharing problem. Theorem 5.1 points out that one way is to restrict the control spaces of the agents, as they directly influence the equilibrium properties. In addition it should be the planners concern that the game rules are fair in the sense that these constitute an idea of equity by which the players are granted equal ex ante opportunities. Basically, we aim at classifying dynamics that satisfy the following properties:

\section{Equilibrium Utility Lowerbound (EUL)}

Consider a Markovian Nash equilibrium $\psi$ in the game $\Gamma_{h, c}\left(x^{0}, t^{0}\right)$ with end-state $x^{*}$. Such $\psi$ is supposed to be cost-based, that is, the corresponding equilibrium utility should meet the following lowerbound

$$
\mathcal{J}_{i}(\psi) \geq F_{i}\left(x_{i}^{*}\right)-F_{i}\left(x_{i}^{0}\right)-\left(x_{i}^{*}-x_{i}^{0}\right) \cdot \max _{x \in\left[x^{0}, x^{*}\right]} D_{i} c(x) .
$$

\section{Free disposal (FD)}

Each agent may obtain any desired amount of his good by an appropriate choice of his controls, independent from the other players' strategies.

\section{Scale invariance}

Change of unit does not change utility in equilibrium in the following sense. Let $s$ : $\mathbb{R}_{+}^{N} \rightarrow \mathbb{R}_{+}^{N}$ be a positive transformation of scales. Denote by $\Gamma_{h, c}^{s}\left(x^{0}, t^{0}\right)$ the game $\Gamma_{h, \cos ^{-1}}\left(s\left(x^{0}\right), t^{0}\right)$ out of $\Gamma_{h, c}\left(x^{0}, t^{0}\right)$ such that the utility for player $i$ along a finite and increasing path $x$ with $x\left(t^{0}\right)=s\left(x^{0}\right)$ is given by

$$
J_{i}^{s}(x)=\int_{t^{0}}^{\infty}\left(\left(F_{i} \circ s_{i}^{-1}\right)^{\prime}\left(x_{i}(t)\right)-D_{i}\left(c \circ s^{-1}\right)(x(t))\right) \dot{x}_{i}(t) \mathrm{d} t .
$$

Note that indeed $c \circ s^{-1}$ is a strictly convex function. Compare the Markov Nash equilibria and corresponding utility profiles of the two-player games $\Gamma_{g, c}\left(x^{0}, t^{0}\right)$ and $\Gamma_{g, c}^{s}\left(x^{0}, t^{0}\right)$, that are related via the positive transform of scales $s$. Suppose that in each Markovian Nash equilibrium of $\Gamma_{g, c}\left(x^{0}, t^{0}\right)$ the utilities are uniquely determined by the profile $\left(\mathcal{J}_{1}^{*}, \mathcal{J}_{2}^{*}\right)$. Then the profile of utilities in each Markovian Nash equilibrium in the scaled game $\Gamma_{g, c}^{s}\left(x^{0}, t^{0}\right)$ equals $\left(\mathcal{J}_{1}^{*}, \mathcal{J}_{2}^{*}\right)$ as well.

Theorem 6.1 Consider the class of autonomous production games with the properties SI, EUL, and FD. Each such game has a unique Markov perfect Nash equilibrium iff this equilibrium is equivalent to that in the game with $h$ such that $h_{i}^{c}(x)=\left(D_{i} c(x)\right)^{-1}$. 
Proof. Let $c$ be a given cost function in $\mathcal{C}$, and $x^{*}$ the solution to the system of differential equations

$$
\left\{\begin{array}{l}
\dot{x}(t)=h_{i}^{c}(x(t)) \\
x(0)=\mathbf{0}
\end{array}\right.
$$

Assume for the moment that $\lim _{t \rightarrow \infty} x_{i}^{*}=\infty$ for both $i=1$ as well as $i=2$. (It is here where we need something like free disposal to close the proof.) Take any output vector $q^{*} \in \mathbb{R}_{+}^{2}$ and consider the path $p^{*}: t \mapsto x^{*}(t) \wedge q^{*}$. Given $p^{*}$ define

$$
\left\{\begin{array}{l}
t_{i}^{*}:=\min \left\{t \mid p_{i}^{*}(t) \geq q_{i}^{*}\right\}, \\
f_{i}\left(q_{i}\right):=\max \left\{D_{i} c\left(p^{*}\left(t_{i}^{*}\right)\right)+q_{i}^{*}-q_{i}, 0\right\} \text { for all } q_{i} \in \mathbb{R}_{+} .
\end{array}\right.
$$

Then $q^{*}$ is the end-state in equilibrium for the equilibrium profile $\psi^{*}$ with equilibrium path $p^{*}$. Hence players have equilibrium utilities

$$
\mathcal{J}_{i}\left(\psi^{*}\right)=F_{i}\left(q_{i}^{*}\right)-\int_{0}^{t_{i}^{*}} D_{i} c\left(p^{*}(t)\right)\left(p_{i}^{*}\right)^{\prime}(t) \mathrm{d} t .
$$

Note that in any game with end-state $q^{*}$ the equilibrium trajectory is the same in any game $\Gamma_{h, c}(\mathbf{0}, 0)$, and in particular that induced by $p^{*}$. We conclude that each $q^{*} \in \mathbb{R}_{+}^{*}$ can be reached as end-state in equilibrium and that associated are unique cost shares $\mu\left(q^{*}, c\right)$ defined by

$$
\mu_{i}\left(q^{*}, c\right)=\int_{0}^{t_{i}^{*}} D_{i} c\left(p^{*}(t)\right)\left(p_{i}^{*}\right)^{\prime}(t) \mathrm{d} t
$$

Note that by varying over all profiles $\left(q^{*}, c\right)$ the above equation (11) defines a cost sharing mechanism. Below we show that given the assumptions (b)-(d) that this cost sharing mechanism equals the Moulin-Shenker rule $\mu^{\mathrm{MS}}$.

As the utilities of the players are unaffected by changes of unit scale, this mechanism must be scale invariant. Moreover, the lower bound on utility implies that $\mu$ satisfies UPP. Then by UPP and continuity of $h^{c}$ it holds that $D_{1} c(x)=D_{2} c(x)$ implies $h_{1}^{c}(x)=h_{2}^{c}(x)$. Otherwise we can construct a subgame according to which the lowerbound on utility is not satisfied in equilibrium, as the induced cost shares are not consistent with UPP.

Define the change of scale $s(x)=\left(D_{1} c\left(x^{0}\right) x_{1}, D_{2} c\left(x^{0}\right) x_{2}\right)$ and denote by $\bar{x}$ the equilibrium path in the scaled game $\Gamma_{h, c}^{s}\left(x^{0}, t^{0}\right)$. Note that by this particular choice of $s$ we get $D_{i}\left(c \circ s^{-1}\right)\left(s\left(x^{0}\right)\right)=1$ for $i=1,2$. Hence, the lower bound on equilibrium utility in the game $\Gamma_{h, c}^{s}\left(x^{0}, t^{0}\right)$ together with continuity of the functions in the family $h$ imply

$$
h_{1}^{\cos ^{-1}}\left(s\left(x^{0}\right)\right)=h_{2}^{\cos ^{-1}}\left(s\left(x^{0}\right)\right) .
$$

Moreover, the utility along the equilibrium path $\bar{x}$ in $\Gamma_{h, c}^{s}\left(x^{0}, t^{0}\right)$ equals $J^{s}(\bar{x})=J\left(s^{-1} \circ \bar{x}\right)$. Note that from (12) we may conclude that

$$
\frac{\left(s^{-1} \circ \bar{x}\right)_{1}^{\prime}\left(t^{0}\right)}{\left(s^{-1} \circ \bar{x}\right)_{2}^{\prime}\left(t^{0}\right)}=\frac{h_{1}^{\cos ^{-1}}\left(s\left(x^{0}\right)\right) / D_{1} c\left(x^{0}\right)}{h_{2}^{\cos ^{-1}}\left(s\left(x^{0}\right)\right) / D_{1} c\left(x^{0}\right)}=\frac{D_{2} c\left(x^{0}\right)}{D_{1} c\left(x^{0}\right)} .
$$


Now suppose that the direction of the paths $s^{-1} \circ \bar{x}$ and $p^{*}$ in $t=t^{0}$ are not equal, or, equivalently, by (13),

$$
\frac{h_{1}^{c}\left(x^{0}\right)}{h_{2}^{c}\left(x^{0}\right)} \neq \frac{D_{2} c\left(x^{0}\right)}{D_{1} c\left(x^{0}\right)} .
$$

Assume without loss of generality that $h_{1}^{c}\left(x^{0}\right) D_{1} c\left(x^{0}\right)<h_{2}^{c}\left(x^{0}\right) D_{2} c\left(x^{0}\right)$ and assume moreover that the equilibrium end-state $\bar{x}(\bar{t})$ is close enough to $s\left(x^{0}\right)$ to ensure that

$$
\frac{h_{1}^{c}(x)}{h_{2}^{c}(x)}<\frac{D_{2} c\left(x^{0}\right)}{D_{1} c\left(x^{0}\right)} \text { for all } x \in\left[x^{0}, s^{-1}(\bar{x}(\bar{t}))\right] .
$$

Note that we used continuity of $h^{c}$ here. By Theorem 5.1 the equilibrium utility for player 1 is met when both players use maximal controls. Let $x^{*}$ be the corresponding equilibrium path in $\Gamma_{h, c}\left(x^{0}, t^{0}\right)$. Define the path $\tilde{x}$ by $\tilde{x}(t)=s^{-1}(\bar{x}(\bar{t})) \wedge x^{*}(t)$. Then a reasoning similar to that in the proof of Theorem 5.1 shows that

$$
J_{1}\left(x^{*}\right) \geq J_{1}(\tilde{x})>J_{1}\left(s^{-1} \circ \bar{x}\right)=J_{1}^{s}(\bar{x}) .
$$

In particular this shows that equilibrium utility has changed by scaling through $s$, which contradicts property (b). So instead of (14) we must have equality and by variation of $x^{0}$ we obtain

$$
\frac{h_{1}^{c}(x)}{h_{2}^{c}(x)}=\frac{D_{2} c(x)}{D_{1} c(x)} \text { for all } x \in \operatorname{int}\left(R_{1} \cap R_{2}\right) .
$$

Therefore, in $\operatorname{int}\left(R_{1} \cap R_{2}\right)$ the costs along the equilibrium path are equally shared. But then the induced cost sharing mechanism $\mu$ is self-consistent as each Markov perfect Nash equilbrium is time-consistent. Hence, we proved that $\mu$ has all the properties enlisted in Theorem 4.4 and must therefore equal $\mu^{\mathrm{MS}}$. In turn this implies that the equilibrium trajectories are in fact the image of the Moulin-Shenker path. Finally, each such equilibrium is represented by the equilibrium in the game where we set $h_{i}^{c}(x)=D_{i} c(x)^{-1}$ for all $x \in \mathbb{R}_{+}^{N}, c \in \mathcal{C}$.

\section{Concluding Remark}

The above analysis is merely concerned with autonomous differential games without any time-dependency. Nevertheless, the above analysis does not change much if we would include discount factors $\left\{r_{i}\right\}_{i=1,2}$ such that the utility functions take the form

$$
J_{i}(x)=\int_{t_{0}}^{\infty} e^{-r_{i} t}\left(f_{i}\left(x_{i}(t)-D_{i} c(x(t))\right)\right) \dot{x}_{i} \mathrm{~d} t .
$$

This is due to the fact that the shape of the profitable regions is not influenced by such adaptation. 


\section{ApPENDix}

Proof of Lemma 3.6 Take $c \in \mathcal{C}$, normalized. We will show that there for any $\epsilon>0$ and $t$ small enough

$$
\left|\frac{\mu\left(t e_{N}\right)}{c\left(t e_{N}\right)}-\frac{1}{|N|}\right| \leq \epsilon
$$

By continuity of the partial derivatives of $c$ there is a $t(\epsilon)>0$ such that for all $t \leq t(\epsilon)$ and all $i \in N$ :

$$
1-\frac{\epsilon}{1+\epsilon} \leq D_{i} c\left(t e_{N}\right) \leq 1+\frac{\epsilon}{1+\epsilon} .
$$

Then from these inequalities (16) and (15) we deduce for $|N| \geq 2$ that

$$
\frac{\mu_{i}\left(t e_{N}, c\right)}{c\left(t e_{N}\right.} \leq \frac{t\left(1+\frac{\epsilon}{1+\epsilon}\right)}{c\left(t e_{N}\right)} \leq \frac{t\left(1+\frac{\epsilon}{1+\epsilon}\right)}{|N| t\left(1-\frac{\epsilon}{1+\epsilon}\right)} \leq \frac{1}{|N|}(1+2 \epsilon) \leq \frac{1}{|N|}+\epsilon .
$$

and

$$
\frac{\mu_{i}\left(t e_{N}, c\right)}{c\left(t e_{N}\right.} \geq \frac{t\left(1-\frac{\epsilon}{1+\epsilon}\right)}{c\left(t e_{N}\right)} \geq \frac{t\left(1-\frac{\epsilon}{1+\epsilon}\right)}{|N| t\left(1+\frac{\epsilon}{1+\epsilon}\right)} \geq \frac{1}{|N|}(1-2 \epsilon) \geq \frac{1}{|N|}-\epsilon .
$$

Then, of course, this proves (15). If $|N|=1$ the statement is obviously true by budget balance.

Proof of Lemma 4.3 Let $\mu$ be a continuous cost sharing mechanism that satisfies no exploitation and self consistency. We will define a collection of paths by which $\mu$ is generated. Let $p^{0, c, N}$ be the set $\left\{q \in \mathbb{R}_{+}^{N} \mid \mu_{i}(q, c)=\frac{c(q)}{n}\right.$ for all $\left.i \in N\right\}$. We claim the following:

(i) For each $t \geq 0$ there is exactly one $q \in p^{0, c, N}$ with $c(q)=t$.

(ii) If $q, q^{\prime} \in p^{0, c, N}, q \neq q^{\prime}$ either $q \ll q^{\prime}$ or $q^{\prime} \ll q$.

First we will prove $(i)$. The first step consists of showing that there is at least one such $q$ for all $t \in \mathbb{R}_{+}$. For $t=0$, obviously there is only such $q$ and that is $q=0$. Let $t>0$ and define $A(t)$ to be the isocost surface for $c$ at level $t$. Then $h: A(t) \rightarrow \Delta^{N}$ with $h(q)=q / \sum_{i \in N} q_{i}$ for all $q \in A(t)$ defines a homeomorphism. Denote its continuous inverse by $h^{-1}$. Next define the mapping $g: \Delta^{N} \rightarrow \Delta^{N}$ by $g(q)=t^{-1} \mu\left(h^{-1}(q), c\right)$. Then by continuity of both $q \mapsto \mu(q, c)$ and $h^{-1}$, it follows that $g$ is continuous. Note that for all $q \in \Delta^{N}$ it holds that $\left\{q_{i}=0 \Rightarrow g_{i}(q)=0\right\}$ by no exploitation. We are ready if we prove that there is a $z \in \Delta^{N}$ such that $g_{i}(z)=\frac{1}{n}$ for all $i \in N$. Define $G: \Delta^{N} \rightarrow \Delta^{N}$ by

$$
G_{i}(q)=\frac{q_{i}+\max \left\{\frac{1}{n}-g_{i}(q), 0\right\}}{1+\sum_{j \in N} \max \left\{\frac{1}{n}-g_{j}(q), 0\right\}} \text { for all } i \in N .
$$

We claim that $G(z)=z$ implies $g_{i}(z)=\frac{1}{n}$ for all $i \in N$. Suppose the opposite, $G(z)=z$ while not $g_{i}(z)=\frac{1}{n}$ for all $i \in N$. Then there are $k, l \in N$ such that $g_{k}(z)<\frac{1}{n}<g_{l}(z)$. 
Hence, $\sum_{j \in N} \max \left\{\frac{1}{n}-g_{j}(z), 0\right\}>0$ and therefore $G_{i}(z)<z_{i}+\max \left\{\frac{1}{n}-g_{i}(z), 0\right\}$. For $i=k$ this converts to $G_{k}(z)<z_{k}$, which leads to contradiction.

Observe that $G\left(\Delta^{N}\right) \subset \Delta^{N}$, since by no exploitation $G_{i}(q)>0$, also for the case $q_{i}=0$. But now we are there, since by invoking Brouwer's Theorem we guarantee existence of such a fixed point $z$ for $G$.

Now, we turn to proving uniqueness. Suppose that for $q^{1}, q^{2} \in p^{0, c, N}$ such that $q^{1} \neq q^{2}$, it holds that $c\left(q^{1}\right)=c\left(q^{2}\right)$. Define $q^{*}:=q^{1} \vee q^{2}$. Then, in particular it holds that $q^{*}>q^{1}$ and thus $c\left(q^{*}\right)>c\left(q^{1}\right)$. By self-consistency we have $\mu\left(q^{*}, c\right)=\mu\left(q^{1}, c\right)+\mu\left(q^{*}-q^{1}, c^{q^{1}}\right)$ and $\mu\left(q^{*}, c\right)=\mu\left(q^{2}, c\right)+\mu\left(q^{*}-q^{2}, c^{q^{2}}\right)$. Since $\mu\left(q^{1}, c\right)=\mu\left(q^{2}, c\right)$, it holds that

$$
\mu\left(q^{*}-q^{1}, c^{q^{1}}\right)=\mu\left(q^{*}-q^{2}, c^{q^{2}}\right) .
$$

For each $i \in N$ it holds either $q_{i}^{*}=q_{i}^{1}$ or $q_{i}^{*}=q_{i}^{2}$, so by no exploitation the cost share of agent $i$ is either 0 in the cost sharing problem $\left(q^{*}-q^{1}, c^{q^{1}}\right)$ or in the cost sharing problem $\left(q^{*}-q^{2}, c^{q^{2}}\right)$. But then by equality (17) we get $\mu\left(q^{*}-q^{1}, c^{q^{1}}\right)=0$, and consequently $\mu\left(q^{1}, c\right)=\mu\left(q^{*}, c\right)$. This gives the desired contradiction, since

$$
c\left(q^{1}\right)=\sum_{i \in N} \mu_{i}\left(q^{1}, c\right)=\sum_{i \in N} \mu_{i}\left(q^{*}, c\right)=c\left(q^{*}\right) .
$$

So, this proves our first claim.

Then, a direct consequence of $(i)$ is that each $t \geq 0$ defines a unique bundle $y(t) \in p^{0, c, N}$ such that $c(y(t))=t$. We will prove that the mapping $y: t \mapsto y(t)$ is continuous. Continuity at $t=0$ is obvious. Suppose on the contrary that there is $t^{*}>0$ and a sequence $t_{1}, t_{2}, \ldots$ in $\mathbb{R}_{++}$such that $\lim _{k \rightarrow \infty} t_{k}=t^{*}$, while the sequence $y\left(t_{1}\right), y\left(t_{2}\right), \ldots$ does not converge to $y\left(t^{*}\right)$. Take $\epsilon>0$ such that $B_{\epsilon}:=\left\{z \in \mathbb{R}_{+}^{N} \mid\left\|y\left(t^{*}\right)-z\right\|<\epsilon\right\}$ is contained in $\mathbb{R}_{+}^{N}$, while there is a subsequence $t_{1}^{\prime}, t_{2}^{\prime}, \ldots$ of $t_{1}, t_{2}, \ldots$ such that for each $k \in N, y\left(t_{k}^{\prime}\right) \notin B_{\epsilon}$. Define $r:=\max _{k \in \mathbb{N}} t_{k}^{\prime}$. Then the sequence $y\left(t_{1}^{\prime}\right), y\left(t_{2}^{\prime}\right), \ldots$ is contained in the compact set $\left\{z \in \mathbb{R}_{+}^{N} \mid c(z) \leq r\right\} \backslash B_{\epsilon}$. Hence, there exists a subsequence $t_{1}^{\prime \prime}, t_{2}^{\prime \prime}, \ldots$ of $t_{1}^{\prime}, t_{2}^{\prime}, \ldots$ such that $y\left(t_{1}^{\prime \prime}\right), y\left(t_{2}^{\prime \prime}\right), \ldots$ converges, say to $q$. Observe that $q \neq y\left(t^{*}\right)$. By continuity of $\mu$, it holds for all $i \in N$,

$$
\mu_{i}(q, c)=\lim _{k \rightarrow \infty} \mu_{i}\left(y\left(t_{k}^{\prime \prime}\right), c\right)=\lim _{k \rightarrow \infty} \frac{c\left(y\left(t_{k}^{\prime \prime}\right)\right)}{n}=\lim _{k \rightarrow \infty} \frac{t_{k}^{\prime \prime}}{n}=\frac{t^{*}}{n} .
$$

Consequently, also $q \in p^{0, c, N}$ and $c(q)=t^{*}$, but with $y\left(t^{*}\right)$ as the unique vector satisfying these conditions, we reached a contradiction. So, $y$ is continuous.

We will now prove $(i i)$. Take $q, q^{\prime} \in p^{0, c, N}$ such that $q \neq q^{\prime}$. Then $(i)$ implies $c(q) \neq c\left(q^{\prime}\right)$. Without loss of generality we will assume that $c\left(q^{\prime}\right)<c(q)$. Suppose that not $q^{\prime} \ll q$. By the continuity of $y$, there is a maximal $t^{\prime}<c\left(q^{\prime}\right)$ such that $y\left(t^{\prime}\right) \in[0, q]$. Hence, by self consistency,

$$
\mu(q, c)=\mu\left(y\left(t^{\prime}\right), c\right)+\mu\left(q-y\left(t^{\prime}\right), c^{y\left(t^{\prime}\right)}\right) .
$$


But for all $i \in N$, we have $\mu_{i}(q, c)-\mu_{i}\left(y\left(t^{\prime}\right), c\right)=\frac{1}{n}\left(c(q)-c\left(y\left(t^{\prime}\right)\right)\right)$, and therefore corresponding to $\mu$ all shares in the problem $\left(q-y\left(t^{\prime}\right), c^{y\left(t^{\prime}\right)}\right)$ are equal. However, $t^{\prime}$ is taken such that $\left(q-y\left(t^{\prime}\right)\right)_{i}=0$ for at least one coordinate $i \in N$. Then, by no exploitation, the corresponding cost share of agent $i$ is 0 , hence the corresponding cost shares for the others are also 0. On the other hand, cost shares sum up to the total cost $c^{y\left(t^{\prime}\right)}\left(q-y\left(t^{\prime}\right)\right)$, which equals $c(q)-c\left(y\left(t^{\prime}\right)\right)=c(q)-t^{\prime}$. But recall that $t^{\prime}<c\left(q^{\prime}\right)<c(q)$, which yields

$$
0=\sum_{i \in N} \mu_{i}\left(q-y\left(t^{\prime}\right), c^{y\left(t^{\prime}\right)}\right)=c(q)-t^{\prime}>0
$$

a contradiction. Therefore $q^{\prime} \ll q$, which ascertains the validity of our second claim.

Now $(i)$ together with $(i i)$ show that $p^{0, c, N}$ is the image of a path, which we will denote by $\pi^{0, c, N}$.

We proceed as follows. Define for all $d \in p^{0, c, N}$ and nonempty sets $S \subset N$ the set $p^{d, c, S}$ by

$$
\left\{q \in \mathbb{R}_{+}^{S} \mid \mu_{i}\left(\left(0_{N \backslash S}, q\right), c^{d}\right)=\frac{1}{|S|} c^{d}\left(\left(0_{N \backslash S}, q\right)\right) \text { for all } i \in S\right\} .
$$

Then, essentially by the same reasoning as before, it follows that $p^{d, c, S}$ is the image of a path $\pi^{d, c, S}$. Take again $d^{\prime}$ as element of one of the previously defined sets $p^{d, c, S}$, and let $d^{1}:=d+\left(0_{N \backslash S}, d^{\prime}\right)$. Define for all $S^{1} \subset S, S^{1} \neq \emptyset$, the set $p^{d^{1}, c, S^{1}}$ by

$$
\left\{q \in \mathbb{R}_{+}^{S^{1}} \mid \mu_{i}\left(\left(0_{N \backslash S^{1}}, q\right), c^{d^{1}}\right)=\frac{1}{\left|S^{1}\right|} c^{d^{1}}\left(\left(0_{N \backslash S^{1}}, q\right)\right) \text { for all } i \in S^{1}\right\} .
$$

Again, essentially the same techniques as before show that this is the image of a path for $S^{1}$. In exactly the same way we proceed inductively by defining paths for coalitions of decreasing size. At the end of this procedure there still may be combinations $(d, c, S)$ left for which $\pi^{d, c, S}$ is not defined; for any of those triples we take $\pi^{d, c, S}$ to be an arbitrary path. Then this completes the definition of a path collection $\Pi$.

It is now an easy exercise to show that it constitutes $\mu$, or $\mu=\mu^{\Pi}$. Let $q \in \mathbb{R}_{+}^{N}$. Suppose $q \in \pi^{0, c, N}\left(\mathbb{R}_{+}\right)$. Then according to $\mu^{\Pi}$, costs $c(q)$ are split equally. But recall the definition of $p^{0, c, N}$ which contains $q$, in order to see that the same division is made in case of $\mu$. If $q \notin \pi^{0, c, N}\left(\mathbb{R}_{+}\right)$, then let $t_{1}$ be the first moment that $\pi^{0, c, N}$ meets the demands of the agents $N_{1}$. Let $d^{1}:=\pi^{0, c, N}\left(t^{1}\right)$ and suppose that $\left(q-d^{1}\right)_{N \backslash N_{1}} \in \pi^{d^{1}, c, N \backslash N_{1}}\left(\mathbb{R}_{+}\right)$. First notice that $\mu_{i}\left(d^{1}, c\right)=\frac{1}{n} c\left(d^{1}\right)$ for all $i \in N$. Suppose that the vector of remaining demands $\left(q-d^{1}\right)_{N \backslash N_{1}}$ is on the path for $N \backslash N_{1}, \pi^{d^{1}, c, N \backslash N_{1}}$. Then as a consequence

$$
\mu\left(q-d^{1}, c^{d^{1}}\right)=\frac{1}{\left|N \backslash N_{1}\right|} c^{d^{1}}\left(q-d^{1}\right) .
$$


Thus, by self consistency for all $i \in N \backslash N_{1}$,

$$
\begin{aligned}
\mu(q, c) & =\mu\left(d^{1}, c\right)+\mu\left(q-d^{1}, c^{d^{1}}\right) \\
& =\frac{1}{|N|} c\left(d^{1}\right)+\frac{1}{\left|N \backslash N_{1}\right|} c^{d^{1}}\left(q-d^{1}\right)=\mu^{\Pi}(q, c) .
\end{aligned}
$$

If not $\left(q-d^{1}\right)_{N \backslash N_{1}} \in \pi^{d^{1}, c, N \backslash N_{1}}\left(\mathbb{R}_{+}\right)$, then proceed by following $\pi^{d^{1}, c, N \backslash N_{1}}$ up to the first moment $t_{2}$ that some agents $N_{2} \subset N \backslash N_{1}$ are satisfied with the present production level. Then the previous reasoning can just be replicated until, finally, a point is reached at which the remaining demand bundle is on the corresponding path for the remaining demanders.

Proof of Theorem 4.4 It is clear that on $\mathcal{G}$ the Moulin-Shenker rule obeys all the enlisted principles.

Now suppose that $\mu$ is a cost sharing rule satisfying CONT, SI, NOEXP, SCONS and (5). We will show that $\mu=\mu^{\mathrm{MS}}$ in the following way.

By Lemma 4.3 it follows that $\mu$ is generated by a path collection $\Pi$. Thus $\mu$ satisfies the serial principle according to Lemma ??. There is no unique way to describe $\Pi$; all other path collections resulting from choosing other parametrizations for the paths in $\Pi$ generate $\mu$ as well. Then it suffices to prove that a path collection by which $\mu$ is generated can be chosen such that it equals $\Gamma$, one of the path collections corresponding to the Moulin-Shenker rule. Therefore the theorem will be proved if we show that, starting with an arbitrary path collection $\Pi$ generating $\mu$, for all $(d, c, S) \in \mathbb{R}^{N} \times \mathcal{C} \times \mathcal{P}(N)$ the path $\pi^{d, c, S} \in \Pi$ is equal to $\gamma^{d, c, S}$ up to parametrization.

We claim that there is a parametrization $\bar{\pi}$ of $\pi^{0, c, N}$, which is a solution to the above system of differential equations (7). Then $\bar{\pi}$ must coincide with $\gamma^{0, c, N}$ by uniqueness of the solution.

Then by simple variations the same reasoning shows that all paths of type $\pi^{0, c^{d}, S}$ are equal to $\gamma^{0, c^{d}, S}$ up to parametrization, for all $d \in \mathbb{R}^{N}$ and $S \in \mathcal{P}(N)$.

First, we will show that $\pi^{d, c, S}\left(\mathbb{R}_{+}\right)=\pi^{0, c^{d}, S}\left(\mathbb{R}_{+}\right)$. We need only to consider those profiles $d$, which can actually be produced using the path collection $\Pi$ and the above construction. Suppose we have an inequality instead and that a cost sharing problem $(q, c)$ the path constructed from $\Pi$ reaches the profile $d$ after a specific period.

Then the path $\pi^{d, c, S}$ is used in the above construction from the very moment where all agents in $N \backslash S$ are satisfied with production. Still, their individual completion times for production may differ. At least the agents in $S$ will have made equal contributions to the procedure of equally splitting incremental costs for raising production levels, since they have not completed yet. So we have $\mu_{i}(d, c)=\mu_{j}(d, c)$ for all $i, j \in S$.

Let $q \in d+\left(\pi^{d, c, S}\left(\mathbb{R}_{+}\right), 0_{N \backslash S}\right)$ and assume that $q \notin d+\left(\pi^{0, c^{d}, S}\left(\mathbb{R}_{+}\right), 0_{N \backslash S}\right)$. Then also the 
payments for the cost sharing problem $(q, c)$ according to the rule $\mu$ are the same for the individual agents in $S$, or $\mu_{i}(q, c)=\mu_{j}(q, c)$ for all $i, j \in S$. Applying SCONS gives

$$
\mu_{i}(q, c)=\mu_{i}(d, c)+\mu_{i}\left(q-d, c^{d}\right) \text { for } i \in S .
$$

So, actually the cost shares for the reduced cost sharing problem $\left(q-d, c^{d}\right)$ must be equal for the agents in $S, \mu_{i}\left(q-d, c^{d}\right)=\mu_{j}\left(q-d, c^{d}\right)$ for all $i, j \in S$. Recall the construction of sharing the cost in the cost sharing problem $\left(q-d, c^{d}\right)$. First the production plan $\pi^{0, c^{d}, S}$ is used in order to define the first production level $y$ at which a set $S^{\prime}$ of agents in $S$ are satisfied with the production so far. By assumption, however, this cannot be the profile $q-d$. So $y<q-d$. Now the incremental cost for bringing production from level 0 up to $y$ are split equally among the members of $S$. Then the procedure continues in order to divide the remaining costs $c^{d}(q-d)-c^{d}(y)$ among the agents $S \backslash S^{\prime}$, which is a nonempty set. Because $c^{d}(q-d)-c^{d}(y)>0$ this means that there is at least one agent in $S \backslash S^{\prime}$ that pays more than any of the agents in $S^{\prime}$. So there are differences in cost shares of agents in $S$ which gives the desired contradiction.

In the proof we roughly distinguish between four steps.

Step 1: The properties SP, SI and (5) allow us to specify $D \pi^{0, c, N}$ up to multiplication with a scalar $y$ under the assumptions of existence of $D \pi^{0, c, N}$ and $D \pi^{0, c, N} \gg 0$. We claim that for all $i \in N$

$$
D_{i} \pi^{0, c, N}(0)=\frac{y}{D_{i} c(0)} .
$$

This is proved as follows. Suppose all partial derivatives of $\pi^{0, c, N}$ are strictly positive. Define the scale transformation $f: \mathbb{R}^{N} \rightarrow \mathbb{R}^{N}$ by

$$
f_{i}(u)=\frac{u_{i}}{D_{i} c(0)} \text { for all } i \in N, u \in \mathbb{R}^{N} .
$$

Then by scale invariance for any $q \in \mathbb{R}^{N}$ the problem $(q, c)$ is equivalent with $\left(f^{-1}(q), c \circ f\right)$. But the latter one is normalized in the sense that for all $i, D_{i}(\operatorname{cof})(0)=D_{i} c(0) D_{i} f(0)=1$. Then by the serial principle and (5) we have for all $i, j \in N$,

$$
\lim _{r \downarrow 0} \frac{\mu_{i}\left(r e_{N}, c \circ f\right)}{\mu_{j}\left(r e_{N}, c \circ f\right)}=1 \text {. }
$$

But this will only be the case if for all $i, j \in N$

$$
\lim _{t \downarrow 0} \frac{\pi_{i}^{0, c \circ f, N}(t)}{\pi_{j}^{0, c \circ f, N}(t)}=1 .
$$

Then by Lemma 4.2 for all $i, j \in N$

$$
\lim _{t \downarrow 0} \frac{f_{i}\left(\pi^{0, c, N}(t)\right)}{f_{j}\left(\pi^{0, c, N}(t)\right)}=1 .
$$


Thus as a result

$$
\lim _{t \downarrow 0} \frac{\left(\pi^{0, c, N}\right)_{i}(t)}{\left(\pi^{0, c, N}\right)_{j}(t)}=\frac{D_{j} c(0)}{D_{i} c(0)}
$$

It is not difficult to prove the following. Let $h, g: \mathbb{R}_{+} \rightarrow \mathbb{R}_{+}$be continuous mappings for which $h^{\prime}(0)$ and $g^{\prime}(0)$ exist at 0 , with $g>0$ on $(0, \infty), g^{\prime}(0)>0$, we have for $\alpha \in \mathbb{R}_{+}$,

$$
\lim _{t \downarrow 0} \frac{h(t)}{g(t)}=\alpha \Longrightarrow h^{\prime}(0)=\alpha g^{\prime}(0) \text {. }
$$

Then this makes clear that by our regularity assumptions on $D \pi^{0, c, N}(0)$ for all $i, j \in N$,

$$
\frac{D_{i} \pi^{0, c, N}(0)}{D_{j} \pi^{0, c, N}(0)}=\frac{D_{j} c(0)}{D_{i} c(0)}
$$

By taking $y$ such that $D_{1} \pi^{0, c, N}(0)=\frac{y}{D_{1} c(0)}$ we prove our claim.

For $q \in \mathbb{R}^{N}$ we define $t_{q}:=\operatorname{argmin}\left\{\pi^{0, c, N}(t) \geq q\right\}$. Then $t_{q}$ stands for the first moment that $\pi^{0, c, N}$ reaches the boundary of the cube $\left\{u \in \mathbb{R}^{N} \mid u \leq q\right\}$.

Step 2: Take $d \in \pi^{0, c, N}\left(\mathbb{R}_{+}\right), d \neq 0$. Note that $d$ is a demand profile for which $\mu$ determines equal cost shares. Assume now that $D \pi^{0, c^{d}, N}(0)$ exists and $D \pi^{0, c^{d}, N}(0) \gg 0$. We claim that there is a $y \in \mathbb{R}_{+}$such that for all $i \in N$

$$
D_{i} \pi^{0, c, N}\left(t_{d}\right)=\frac{y}{D_{i} c\left(\pi^{0, c, N}\left(t_{d}\right)\right)} .
$$

Essentially this is proved with the techniques from Step 1 together with the property SCONS. Applying Step 1 for $c^{d}$ instead of $c$ immediately provides us with a $y \in \mathbb{R}_{+}$such that for all $i \in N$

$$
D_{i} \pi^{0, c^{d}, N}(0)=\frac{y}{D_{i} c^{d}(0)}=\frac{y}{D_{i} c(d)}=\frac{y}{D_{i} c\left(\pi^{0, c, N}\left(t_{d}\right)\right)} .
$$

On the other hand we find another expression for $D_{i} \pi^{0, c^{d}, N}(0)$ by the relation between $\pi^{0, c^{d}, N}$ and $\pi^{0, c, N}$. By SCONS and the fact that $\mu_{i}(d, c)=\frac{c(d)}{|N|}$, we have for all $d^{\prime} \geq$ $d, d^{\prime} \in \pi^{0, c, N}\left(\mathbb{R}_{+}\right), i \in N$

$$
\mu_{i}\left(d^{\prime}, c\right)=\frac{c(d)}{|N|}+\mu_{i}\left(d^{\prime}-d, c^{d}\right)
$$

Since $d^{\prime}$ is also a demand profile for which $\mu$ determines equal cost shares, it holds for all $i \in N$

$$
\mu_{i}\left(d^{\prime}-d, c^{d}\right)=\frac{c\left(d^{\prime}\right)-c(d)}{|N|} .
$$

But $\mu_{i}\left(d^{\prime}-d, c^{d}\right)=\mu_{j}\left(d^{\prime}-d, c^{d}\right)$ for all $i, j \in N$ if and only if the first splitting point for the problem $\left(d^{\prime}-d, c^{d}\right)$ is $d^{\prime}-d$, or equivalently $d^{\prime}-d \in \pi^{0, c^{d}, N}\left(\mathbb{R}_{+}\right)$. So $d^{\prime} \in \pi^{0, c, N}\left(\mathbb{R}_{+}\right)$if 
and only if $d^{\prime} \in \pi^{0, c^{d}, N}\left(\mathbb{R}_{+}\right)+d$. This in turn implies $\pi^{0, c^{d}, N}\left(\mathbb{R}_{+}\right)=\pi^{0, c, N}\left(\left[t_{d}, \infty\right)\right)$. Since only the images of the paths matter we may assume that $\pi^{0, c^{d}, N}(t)=\pi^{0, c, N}\left(t_{d}+t\right)-d$ for all $t \in \mathbb{R}_{+}$. But this gives for $i \in N, D_{i} \pi^{0, c^{d}, N}(0)=D_{i} \pi^{0, c, N}\left(t_{d}\right)$ and together with equality (18),

$$
D_{i} \pi^{0, c, N}\left(t_{d}\right)=\frac{y}{D_{i} c\left(\pi^{0, c, N}\left(t_{d}\right)\right)} .
$$

Step 3: For almost every $t \in \mathbb{R}_{+}$, there is a $y \in \mathbb{R}_{+}$with

$$
D_{i} \pi^{0, c, N}(t)=\frac{y}{D_{i} c\left(\pi^{0, c, N}(t)\right)} .
$$

The mapping $\pi^{0, c, N}$ is monotonically increasing and therefore differentiable almost everywhere. If only $D \pi^{0, c, N} \gg 0$ almost everywhere, then we are done: the result from Step 2 applies for almost every $d \in \pi^{0, c, N}\left(\mathbb{R}_{+}\right)$, which in turn implies (21).

Let

$$
\tilde{\pi}:=\pi^{0, c, N} \circ\left(c \circ \pi^{0, c, N}\right)^{-1} .
$$

Then $\tilde{\pi}$ is a parametrization of $\pi^{0, c, N}$ by the costs; for each $t \in \mathbb{R}_{+}$it holds that $c(\tilde{\pi}(t))=t$. Take $t \in \mathbb{R}_{+}$and $h>0$. Then,

$$
\begin{aligned}
\left\|\frac{\tilde{\pi}(t+h)-\tilde{\pi}(t))}{h}\right\| & \geq|N| \frac{c(\tilde{\pi}(t+h))-c(\tilde{\pi}(t))}{h b(c)} \\
& =|N| \frac{h}{h b(c)}=|N| b(c)^{-1}>0 .
\end{aligned}
$$

This implies that whenever $\tilde{\pi}$ is differentiable at $t$, then $\tilde{\pi}^{\prime}(t) \gg 0$. But consequently $\tilde{\pi}^{\prime} \gg 0$ almost everywhere, since it is a monotonically increasing function. There is only one possibility, and that is $D \pi^{0, c, N} \gg 0$ almost everywhere. This proves our claim.

Step 4: The last part of the proof is of rather technical nature. We will show now that the above $\tilde{\pi}$ can be used to define the proper parametrization of $\pi^{0, c, N}$ that we are looking for.

Note, that given the fact that $\pi^{0, c, N}$ is monotonically increasing we have for almost all $t \in \mathbb{R}_{+}$:

$(i): \pi^{0, c, N}$ is differentiable at $\left(c \circ \pi^{0, c, N}\right)^{-1}(t)$ and

$$
D \pi^{0, c, N}\left(\left(c \circ \pi^{0, c, N}\right)^{-1}(t)\right)>0 .
$$

(ii): $c \circ \pi^{0, c, N}$ is differentiable at $\left(c \circ \pi^{0, c, N}\right)^{-1}(t)$ and

$$
\left(c \circ \pi^{0, c, N}\right)^{\prime}\left(\left(c \circ \pi^{0, c, N}\right)^{-1}(t)\right)>0 .
$$

So for the parametrization $\tilde{\pi}$ of $\pi^{0, c, N}$, defined above by (22), the following equality holds almost everywhere, for all $i \in N$ 


$$
D_{i} \tilde{\pi}(t)=\frac{D_{i} \pi^{0, c, N}\left(\left(c \circ \pi^{0, c, N}\right)^{-1}(t)\right)}{\left(c \circ \pi^{0, c, N}\right)^{\prime}\left(\left(c \circ \pi^{0, c, N}\right)^{-1}(t)\right)}=\frac{1}{|N|} \frac{1}{D_{i} c(\tilde{\pi}(t))} .
$$

Consider the curve $\bar{\pi}:=\tilde{\pi} \circ \varphi$, where $\varphi(t)=|N| t$ for all $t \in \mathbb{R}_{+}$. Then $\bar{\pi}$ is a parametrization of $\pi^{0, c, N}$ for which for almost all $t \in \mathbb{R}_{+}$it holds that for all $i \in N$

$$
D_{i} \bar{\pi}(t)=\frac{1}{D_{i} c(\bar{\pi}(t))} .
$$

If we can show that this equality holds for all $t \in \mathbb{R}_{+}$, then we are done. Since then we showed that $\bar{\pi}$ is actually the parametrization of $\pi^{0, c, N}$ that we were looking for, because $\bar{\pi}=\gamma^{0, c, N}$. The mapping $\bar{\pi}$ is Lipschitz continuous: for all $t_{1}, t_{2} \in \mathbb{R}_{+}$,

$$
\begin{aligned}
\left\|\bar{\pi}\left(t_{1}\right)-\bar{\pi}\left(t_{2}\right)\right\| & =\left\|\tilde{\pi}\left(|N| t_{1}\right)-\tilde{\pi}\left(|N| t_{2}\right)\right\| \\
& \leq a(c)^{-1}\left|c\left(\tilde{\pi}\left(|N| t_{1}\right)\right)-c\left(\tilde{\pi}\left(|N| t_{2}\right)\right)\right| \\
& =|N| a(c)^{-1}\left|t_{1}-t_{2}\right| .
\end{aligned}
$$

So $\bar{\pi}$ is absolutely continuous and therefore, for all $i \in N$ and $t \in \mathbb{R}_{+}$,

$$
\bar{\pi}(t)=\int_{0}^{t} D_{i} \bar{\pi}(s) d s=\int_{0}^{t} D_{i} c(\bar{\pi}(s))^{-1} d s .
$$

By the continuity of the mapping $s \mapsto D_{i} c(\bar{\pi}(s))^{-1}$, it follows that $\bar{\pi}$ is differentiable and for all $i \in N$

$$
D_{i} \bar{\pi}(t)=\frac{1}{D_{i} c(\bar{\pi}(t))} \text { for all } t \in \mathbb{R}_{+} .
$$

But then $\bar{\pi}$ is a solution of the system of differential equations that determines the MoulinShenker path $\gamma^{0, c, N}$. By uniqueness of the solution $\bar{\pi}$ must coincide with $\gamma^{0, c, N}$. This proves our claim that $\pi^{0, c, N}$ has the same image as $\gamma^{0, c, N}$.

\section{REFERENCES}

1. R. J. Aumann and L. S. Shapley, Values of non-atomic games, Princeton University Press, Princeton, New Jersey, U.S.A., 1974.

2. L. Billera and D. Heath, Allocation of shared costs: a set of axioms yielding a unique procedure, Mathematics of Operations Research 7 (1982), 32-39.

3. E.A. Coddington and N. Levinson, Theory of ordinary differential equations, McGraw-Hill, New York, U.S.A., 1955.

4. E. Dockner, S. Jorgensen, N. Van Long, and G. Sorger, Differential games in economics and management science, Cambridge University Press, Cambridge, UK, 2000.

5. E. Friedman and H. Moulin, Three methods to share joint costs or surplus, Journal of Economic Theory 87 (1999), 275-312.

6. V. Kolpin, Coalition equitable cost sharing of multi-service facilities, Mimeo, University of Oregon, Eugene, Oregon, U.S.A., 1994. 
7. __ Equitable nonlinear price regulation: an alternative approach to serial cost sharing, Games and Economic Behavior (1998), 61-83.

8. V. Kolpin and D. Wilbur, Bayesian serial cost sharing, forthcoming in Mathematical Social Sciences, 2005.

9. F. Maniquet, Allocation rules for a commonly owned technology: the average cost lower bound, Journal of Economic Theory 69 (1996), 490-507.

10. H. Moulin, Cost sharing under increasing returns: a comparison of simple mechanisms, Games and Economic Behavior 13 (1996), 225-251.

11. H. Moulin, Axiomatic cost and surplus-sharing, Handbook of Social Choice and Welfare, Volume 1 (Sen Arrow and Suzumura, eds.), North Holland, Elsevier, Amsterdam, 2002, pp. 289-354.

12. H. Moulin and S. Shenker, Average cost pricing versus serial cost sharing: an axiomatic comparison, Journal of Economic Theory 64 (1992), 178-201.

13. H. Moulin and S. Shenker, Serial cost sharing, Econometrica 60 (1992), 1009-1037.

14. H Moulin and S. Shenker, Average cost pricing versus serial cost sharing; an axiomatic comparison, Journal of Economic Theory 64 (1994), 178-201.

15. M. Shubik, Incentives, decentralized control, the assignment of joint cost, and internal pricing, Management Science 8 (1962), 325-343.

16. Y. Sprumont, Ordinal cost sharing, Journal of Economic Theory 81 (1998), 126-162.

17. W. Thomson, Axiomatic analysis and game-theoretic analysis of bankruptcy and taxation problems: a survey, Mathematical Social Sciences (2003).

18. E. Yanovskaya, Serial cost sharing, Mimeo, Russian Academy of Sciences, St. Petersburg, Russia, 2004.

19. H. P. Young, Cost allocation: Methods, principles, applications, North-Holland, Amsterdam, The Netherlands, 1985.

20. _ Cost allocation, Handbook of Game Theory II (R.J. Aumann and S. Hart, eds.), Elsevier, Amsterdam, The Netherlands, 1994, pp. 1193-1235. 